\title{
Open-air Unmovable Cultural Relics Health Trend Prediction
}

\author{
Xiaohong Zhang ${ }^{1,2, a *}$, Huiqin Wang ${ }^{3, b}$, Zhan Wang ${ }^{4,5, c}$, Tao Ma ${ }^{4,5}$, Qi Shang ${ }^{3}$, \\ Wenhan $\mathrm{Li}^{2}$
}

\author{
${ }^{1}$ School of Management,Xi'an University Of Architecture And Technology, Xi'an 710055, \\ China; \\ ${ }^{2}$ School of Communication\&Information Engineering,Xi'an University of \\ Science\&Technology,Xi'an 710054, China \\ ${ }^{3}$ School of Information\&Control Engineering,Xi'an University of Architecture\& \\ Technology ,Xi'an 710055,China \\ ${ }^{4}$ Shaanxi Institute of Heritage Conversation and Restoreation, Xi'an 710075,China \\ ${ }^{5}$ Masonry Quality State Administration of Cultural Heritage, Xi'an 710075,China \\ ${ }^{6}$ School of Economics and Management,Fuzhou University,Fuzhou 350116,China \\ a447973560@qq.com, bwhq463@263.net, csigu@foxmail.com
}

\begin{abstract}
Keywords: cultural relic conservation;health management;relic health management;the internet of things;grey model;grey predication

Abstract. Perennially suffering weathering erosion and man-made damage,open-air unmovable cultural relics' preservation environment is very serious.By taking China Tang Shunling as research object and adopting grey model theory,because of the relic monitoring data with the characteristics of small sample and s-type,the paper builds the $\operatorname{GM}(1,1)$ model and the Verhulst model in analysing and predicting the development trend of cultural relic's cracks. Experimental results show that the average relative errors of forecast are $6.23 \%$ and $2.36 \%$ respectively, which provide a basis for the relic health quantitative analysis and research.Finally, the cultural relic health management platform is designed and implemented by JSP and SQL Server, which realizes the health monitoring data collection, pre-processing and forecasting of the cultural relic health.
\end{abstract}

\section{Introduction}

China is rich in unmovable cultural relics resources, which has great value. Immovable cultural relics kept in the wild or harsh environment perennially, facing sun, wind, earthquakes, floods, theft and other dangerous ruins.Because of the worried cultural relics health, cultural relics protection theories and methods have become the core of cultural relics conservation work.

In the Twelfth Five Year Plan, China Cultural Relics Bureau clearly stated the "preventive" protection philosophy and accurate protection plan from the "prevention, management, maintenance,

management" aspects, proposed to maximize suppression or mitigation of environmental factors on cultural relics material's damaging and to maintain the health status of cultural relics ${ }^{[1]}$. Cultural relics health management analyzes the current state whether them decline or deviate or not,diagnostics predictively the future health state or remaining life of cultural relics based on the system's current or past performance, and makes some appropriate recovery and decisions to extend the remaining life of cultural relics.

Therefore, this paper the paper puts forward the health management methods.On this basis of data ananlysis, because of the monitoring data with the characteristics of small sample and s-type,the paper builds the GM(1,1) model and the Verhulst model in analysing and predicting the development trend of cultural relic's cracks by taking China Tang Shunling as research object,providing the theoretical basis for cultural relics health risk prediction. 


\section{Related Research and Analysis}

It has become a necessary means of preventively protecting cultural relics that monitor cultural relics and environment by using non-destructive monitoring technology.In general, health analysis method is mainly divided into two categories:based on models and based on data-driven ${ }^{[2]}$.

Model-based approach has to establish a mathematical model about monitor system, associating the model with physical processes. This method makes the use of advanced algorithm to estimate model parameters,getting the residuals between model parameter estimation and health model parameters as health diagnostic scalar ${ }^{[3,4]}$. Data-driven health estimation method is a method of data mining or machine learning. The data is collected in accordance with a certain period during the data-driven forecasting process, calculating the associated health feature from the raw data by different feature extraction. Data-driven approach bases on the advanced integration policies in order to combine the operating mode information with the output of classifier,producing a fusion of health status assessment $^{[5,6]}$.

\section{Cultural relics Disease predictive trends based on Grey System Theory}

Traditional forecasting method based on the priori knowledge and statistical methods start with probability distribution to study the statistical law, and the modeling process relies on a large sample of the original data. Grey system theory takes the uncertain system, "some of the information is known, some information is" unknown"and the"small sample" , "poor information" ,as the study object. Gray forecast regards observed data more as a gray process or a gray variable changed over time than as a random process, and establishes the model corresponding to differential equation ,making the forecast later through accumulation and reduction cumulatively to whiten the gray variable ${ }^{[7]}$.

\section{GM (1,1) Prediction Model}

Order $X^{(0)}$ as the original sequence in $\mathrm{GM}(1,1)$ model:The data series is $X^{(0)}=\left(x^{(0)}(1), x^{(0)}(2), \ldots, x^{(0)}(n)\right), X^{(1)}=\left(x^{(1)}(1), x^{(1)}(2), \ldots, x^{(1)}(n)\right)$ is named 1-AGO sequence of $X^{(0)}$, and

$$
x^{(1)}(k)=\sum_{i=1}^{k} x^{(0)}(i), k=1,2, \ldots, n .
$$

The seemingly irregular original sequence will transfer into ascending sequence $X^{(1)}$ in this way, and it is easier to calculate data's law for the character, such as strong law and weak randomness. Then it could do least squares estimation for the equation's parameter by constructing gray differential and whitening equtions, and get $X^{(1)}$.And you can obtain the desired estimated value through regressive calculation $^{[8]}$.

So it takes $Z^{(1)}$ as the mean (MEAN) of $X^{(1)}$ and generates a sequence:

$$
\begin{aligned}
& Z^{(1)}=\left(z^{(1)}(2), z^{(1)}(3), \ldots, z^{(1)}(n)\right) \text {, and } \\
& z^{(1)}(k)=0.5^{x^{(1)}(k)+0.5 .^{x^{(1)}}(k-1) .}
\end{aligned}
$$

And gray differential equation model of GM $(1,1)$ is

$$
x^{(0)}(k)+a z^{(1)}(k)=b,
$$

In which it could use $k=1,2, \ldots$ to get prediction value and prediction sequence for the time response eqution.Then,restore the prediction value of original sequence by using $\mathrm{k}=1,2, \ldots$.

\section{Verhulst Model}

Traditional verhulst model requires the original data with the s-type characteristics. To make monitoring original data had s-type, gray verhulst model does AGO for similar single peak data,and then create verhulst model after gets better S-type ${ }^{[9]}$.

gray verhulst model:

$$
x^{(0)}(\mathrm{k})+\mathrm{az} \mathrm{z}^{(1)}(\mathrm{k})=\mathrm{b}\left(\mathrm{z}^{(1)}(\mathrm{k})\right)^{2}
$$

Among them, the value of parameters $a, b$ are obtained by using least square method, 


$$
\begin{gathered}
\hat{a}=(\mathrm{a}, \mathrm{b})^{\mathrm{T}}=\left(\mathrm{B}^{\mathrm{T}} \mathrm{B}\right)^{-1} \mathrm{~B}^{\mathrm{T}} Y . \\
Y=\left[\begin{array}{l}
x^{(0)}(2) \\
x^{(0)}(3) \\
\cdots \\
x^{(0)}(n)
\end{array}\right], \mathrm{B}=\left[\begin{array}{ll}
-z^{(1)}(2) & \left(z^{(1)}(2)\right)^{2} \\
-z^{(1)}(3) & \left(z^{(1)}(3)\right)^{2} \\
\cdots & \\
-z^{(1)}(n) & \left(z^{(1)}(n)\right)^{2}
\end{array}\right]
\end{gathered}
$$

Time responsive of gray Verhulst model is :

$$
\hat{x}^{(0)}(\mathrm{k})=\frac{a x^{(1)}(0)}{b x^{(1)}(0)-\left(a-\mathrm{bx}^{(1)}(0)\right) \mathrm{e}^{-2 \mathrm{k}}}, k=1,2, \ldots, n,
$$

\section{Health forecasting for Tang Shunling cultural relics}

Tang Shunling is the tomb of Wu Zetian's mother. According to the established prediction model ,getting cracks' month average $(\mathrm{mm})$ for 28-170 X direction in 2013, ray prediction for cultural relics cracks.Establishing gray GM $(1,1)$ forecasting model to verify the fitting accuracy between established GM $(1,1)$ model and verhulst model. Combined with original cracks' data ,for cultural relics cracks is:

$$
\hat{x}^{(0)}(\mathrm{k}+1)=0.0117 \mathrm{e}^{-0.0095 \mathrm{k}}
$$

Establishing verhulst model and abtaining the time response function of gray verhulse model by cultural relics health factors prediction systems is:

$$
\hat{\mathrm{X}}^{(1)}(\mathrm{k}+1)=\frac{-0.002624}{-0.200489-0.057483 e^{-0.257972 k}}
$$

\begin{tabular}{|c|c|c|c|c|c|c|}
\hline \multirow{2}{*}{$\begin{array}{l}\text { Raw data } \\
(\mathrm{cm})\end{array}$} & \multicolumn{3}{|c|}{$\mathrm{GM}(1,1)$} & \multicolumn{3}{|c|}{ Verhulst } \\
\hline & Prediction $(\mathrm{cm})$ & Relative error & MSE & Prediction $(\mathrm{cm})$ & Relative error & MSE \\
\hline 0.012145 & 0.01306 & $7.53 \%$ & \multirow{7}{*}{$\begin{array}{c}6.23 \\
\%\end{array}$} & 0.01287 & $5.97 \%$ & \multirow{7}{*}{$2.63 \%$} \\
\hline 0.012259 & 0.01318 & $7.51 \%$ & & 0.01292 & $5.39 \%$ & \\
\hline 0.012354 & 0.01331 & $7.74 \%$ & & 0.01296 & $4.91 \%$ & \\
\hline 0.012869 & 0.01343 & $4.36 \%$ & & 0.01299 & $0.94 \%$ & \\
\hline 0.012111 & 0.01356 & $12 \%$ & & 0.01301 & $7.42 \%$ & \\
\hline 0.013346 & 0.01369 & $2.58 \%$ & & 0.01303 & $-2.4 \%$ & \\
\hline 0.013554 & 0.01382 & $1.96 \%$ & & 0.01304 & $-3.8 \%$ & \\
\hline
\end{tabular}

The model fitted value and original values for cracks in direction $\mathrm{X}$ is listed in Table 1.

Table 1 cracks prediction error checklist in 2014

By model fitting residual value analysis and fitting error accuracy test , analysing the original data system (28-170 node (crack strain gauge) in the X direction in 2013(mm)) and having gotten that both methods are effective in short-term forecast, and the average relative error is $6.23 \%, 2.63 \%$, both are under $10 \%$. GM $(1,1)$ is feasible for not quickly varied data to predict in short-time volatility,and verhulst model is good than $\operatorname{GM}(1,1)$ in overall fitting if $\operatorname{GM}(1,1)$ has errors in predicting. Verhulst model is suit for predicting in the $\mathrm{X}$ direction in 28-170 node (cracks strain gauge) as shown in Fig.1.

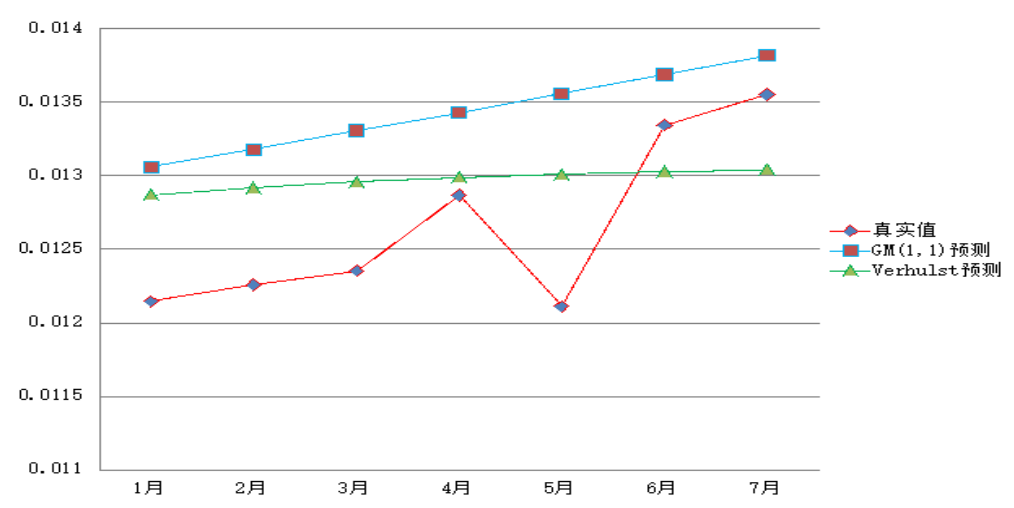

Fig. 1 comparision cracks' predicted values with crack raw data 


\section{Summary}

This article starts with the cultural relics health analysis method based on IOT,taking Tang Shunling in China as object.And it build the $\mathrm{GM}(1,1)$ and verhulst model of cultural relics cracks on the basis of real-time data abtained by IOT.Based on the cracks monitoring data in 2013, it predictsthe data in 2014.And the prediction average relative error between two models are $6.23 \%$ and $2.63 \%$.

However,both models reflected nothing when the cracks in X direction rose quickly between February to May in 2014. In addition, both models prediction maximum relative error is more than $10 \%$, and the prediction value of GM $(1,1)$ model grew rapidly. And the prediction value of verhulst model grew slowly, showing a monotonic curve in trend, which the rate of change is gradually reduced, and the curve toward easing.And verhulst model can not reflect the complexity of cracks data in $\mathrm{X}$ direction to some extent.

\section{Acknowledgements}

This paper is supported by the items: Five-year national ministry of science and technology support project(2012bak14b01) and the returned overseas Chinese science and technology support projects of national ministry of education (K05055).

\section{References}

[1] Tan Zhong-jia, Zhang Zhiqiang, Shi Yan-bin.The Overview of the Health Monitoring Management System[C].2012 International Conference on Medical Physics and Biomedical Engineering,2013:1323-1329.

[2] Dai Jian,Liu Ke. IOT technology based on the safety monitoring and information collection and utilization of traditional wooden construction[J]. Sciences of Conservation and Archaeology, 2011(23)3:39-47.

[3] C. S. Christoforou, L. G. Salmon, G. R. Cass. Air exchange within the buddhist cave temples at YunGang, China[J]. Atmospheric Enuronmenf, 2006,30(23):3995-406.

[4] MyraJ.Giesenatl.Condition assessment and preservation of open-air rock art panels during environmental change. Journal of Cultural Heritage, 2014(15):49-56.

[5] L.G.Salmon,C.S.Chrlstoforou,G.R.Cass.Airborne Pollutants in the buddhist cave temples at the Yungang Grottoes, China[J]. Environmental Science Technology.2004,(28):805 811.

[6] Zhang Yan,Yang Na.The structure modal parameter identification and analysis of environmental stimulus on the ancient timber structure[J]. Journal of Wuhan University of Technology,2010.05:291-295.

[7] Liu Sifeng,Dang Yaoguo,Fang Zhigeng ec.Grey system theory and application[M].Science publication,2010.5.

[8] Zhang Zhenghu,Yuan Mengke,Deng Jianhui.Displacement prediction of slope based on improved Grey-time series time-varying model[J]. Chinese Journal of Rock Mechanics and Engineering, 2014.08:3791-3797.

[9] Xiong Pingping,Dang Yaoguo,Yao Tianxiang,etc.The research on the modeling method of background value optimization in grey Verhulst model[J].Chinese Journal of Management Science, 2012,06:154-159. 